\title{
Metachronous and Synchronous Multiple Primary Carcinomas in an Elderly
}

\author{
Ranim R. Mira ${ }^{\text {a, b, d , Andrea Bial }}{ }^{\mathrm{b}}$, Kellie H. Campbell ${ }^{\mathrm{b}}$, Salman Ali ${ }^{\mathrm{b}}$, \\ Shahad Abdulameer ${ }^{\mathrm{a}}$, Martin J. Gorbien ${ }^{\mathrm{c}}$
}

\begin{abstract}
The prognosis of most cancers has been improved in recent years due to the increased survival of cancer patients, the prolonged lifespan of the general population, and better diagnostic and surgical approaches. Subsequently, the number of patients with multiple primary carcinomas (MPCs) has become greater. In this report, we describe a unique case of a 71-year-old man with five metachronous and synchronous primary malignant tumors. The patient was first diagnosed with synchronous, left posterior tonsillar pillar squamous cell carcinoma (SCC) and right lingual surface of epiglottis SCC in June 2016. Two years later, he presented with three other primary carcinomas within a 3-month time span: right lower lobe lung SCC, right tongue invasive SCC and hepatocellular carcinoma (HCC), consecutively. Investigations revealed no metastases of the primary neoplasms. As the population of older adults with cancer and multimorbidity grows, the therapeutic options usually become limited. On the other hand, understanding the effect of multimorbidity on the care of patients with cancer and developing therapeutic interventions for these elderly patients would be crucial for geriatric care.
\end{abstract}

Keywords: Multiple; Primary; Carcinomas; Malignancies; Cancers; Tumors; Neoplasms; Synchronous; Metachronous; Lung; Liver; Tongue; Tonsil; Oropharynx and epiglottis

\section{Introduction}

Multiple primary carcinomas (MPCs) are defined as two or more primary malignant neoplasms, in which each tumor is not an extension, recurrence, or metastasis of the other malignancies. There are certain criteria for MPCs to be considered as primaries based on what Warren and Gates first described

Manuscript submitted September 17, 2018, accepted September 27, 2018

${ }^{a}$ Loyola University, Maywood, IL, USA

${ }^{\mathrm{b}}$ Edward Hines Jr., VA Hospital, Maywood, IL, USA

${ }^{\mathrm{c}}$ Geriatrics \& Extended Care Center, Edward Hines Jr., VA Hospital, Maywood, IL, USA

${ }^{\mathrm{d} C o r r e s p o n d i n g ~ A u t h o r: ~ R a n i m ~ R . ~ M i r a, ~ L o y o l a ~ U n i v e r s i t y, ~ M a y w o o d, ~ I L ~}$ 60153, USA. Email: ranim_r_mira@yahoo.com

doi: https://doi.org/10.14740/jmc3172 in 1932: 1) Each of the tumors must be malignant; 2) Each of the lesions must exhibit different histopathologic types; and 3) Metastases from the original malignancies must be ruled out $[1,2]$. MPCs can occur in the same site or organ and still be considered separate primaries regardless of whether they are diagnosed at the same or different times as long as the neoplasms have distinctive histologic characteristics [3]. Primaries are divided into simultaneous (synchronous), if diagnosed within six months, and sequential (metachronous) tumors if previous malignant lesions and metachronous ones occur more than 6 months apart. Synchronous tumors are extremely rarer than metachronous tumors. Among patients with MPCs, double cancers are commonly observed, triple cancers occur in $0.5 \%$ of them, and quadruple or quintuple cancers occur in $<$ $0.1 \%$ [4]. The existence of multiple primary cancers in a single patient is relatively uncommon, although improved quality of life, increased survival of cancer patients and a longer lifespan of the general population have expanded the incidence of MPCs. In addition, the rate of developing multiple primaries is noted to rise with increasing age and has a male predominance due to high frequency of prostatic cancer. Therefore, the accurate and early detection and management of this challenging complex condition have become significantly vital $[3,4]$. We herein report a case of patient exhibiting metachronous and synchronous quintuple primary invasive carcinomas, namely a left posterior tonsillar pillar squamous cell carcinoma (SCC), right lingual surface of epiglottis SCC, right lower lobe lung $\mathrm{SCC}$, right tongue $\mathrm{SCC}$ and hepatocellular carcinoma (HCC).

\section{Case Report}

The patient is a 71-year-old male veteran with a past medical history of chronic obstructive pulmonary disease, hypertension, a significant history of tobacco dependence (30 pack-years), alcohol abuse, gastroesophageal reflux disease (GERD), depression and frequent falls. He visited the Department of Oncology because of ulcers in his oropharynx in July 2016. Biopsy was done which revealed two synchronous SCCs of the oropharynx that were treated with wide local excision of the left posterior tonsillar pillar of epiglottis and tonsillectomy. They recurred and were then treated with radiation therapy (RT) in November 2016. Two years later, another primary tumor appeared in the right lateral tongue (T1N0M0) in March 2018, which was treated with partial glossectomy in May 2018. A percutaneous endoscopic gastrostomy (PEG) was in- 


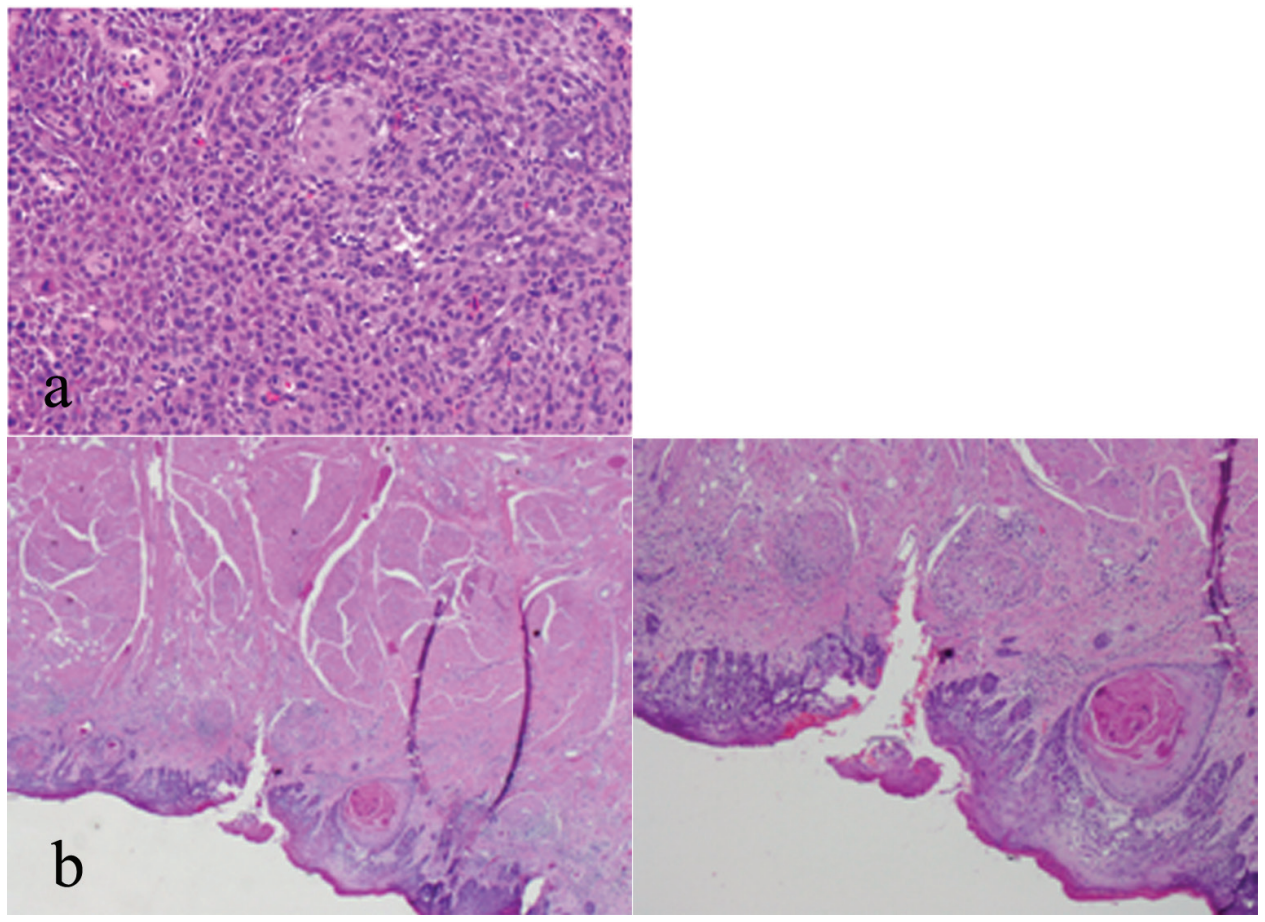

Figure 1. (a) Tonsillar fossa lesion biopsy with high power magnification showing a moderately-differentiated squamous cell carcinoma (SCC) of the left tonsil. (b) Epiglottis biopsy with low and high power magnification showing a moderately-differentiated SCC of the right epiglottis.

serted for tube feeding. He was also diagnosed with SCC of the lung (T2aN0M0) affecting the right upper lobe in March 2018. The patient was not a candidate for bilobectomy, so he underwent RT for SCC of the lung. He only completed four of the eight scheduled sessions until June 2018, as the treatment was complicated by $\mathrm{R}$ lung post-obstructive pneumonia and atelectasis. Furthermore, he was noted to have radiation pneumonitis, so further radiation was held while the patient was being treated with steroids. After that, the patient started complaining of right upper quadrant (RUQ) pain. A computed tomography (CT) scan confirmed cholecystitis with stone in the cystic duct and dilatation of the common bile duct. The patient was treated medically but after 2 weeks he continued to have persistent RUQ abdominal pain. A hepatobiliary iminodiacetic acid (HIDA) scan in May 2018 revealed no evidence of cholecystitis. MRI done in June 2018 showed a large mass in the right lobe of the liver concerning for $\mathrm{HCC}$ based on elevated alpha-fetoprotein (AFP, $495.4 \mathrm{IU} / \mathrm{mL}$ ). He also had very high ferritin and total bilirubin levels compared to his normal levels in 2016. The diagnosis of HCC was confirmed by biopsy. Despite all of the above, the patient is still alive and currently residing in a nursing home.

A macroscopic examination of the resected specimens revealed distinct tumors in his oropharynx. One was on the epiglottis, and the second was found at the left tonsil. Two years later, the third one was found on the right side of his tongue with a size of $1.4 \mathrm{~cm}$.

A histologic examination of the tonsil and epiglottis (Fig. 1a, b) showed a moderately differentiated SCC with invasion into the submucosa, characterized by the presence of cohesive nests and cords of carcinoma with an associated desmoplastic stroma, and apparent keratinization and intercellular bridges in association with the invasive (cohesive) tumor nests were noted. Moderately differentiated keratinizing SCC showed greater nuclear pleomorphism and less differentiation. The cytomorphologic features include moderate nuclear pleomorphism, nuclear hyperchromasia, loss of cellular polarity, increased nuclear/cytoplasmic ratio, and increased mitotic activity that may include atypical mitoses.

The tumor at his tongue (Fig. 2a) with higher magnification shows violation of the basement membrane by scattered tongues of malignant epithelial cells associated with an inflammatory cell infiltrate. Individual dyskeratotic cells are scattered throughout. Glossectomy was done as part of his oncological management and the histological examinations revealed invasive SCC (Fig. 2b).

Endobronchial biopsy of the right lower lobe of his lung mass (Fig. 3a) revealed a keratinizing SCC. Note the presence of keratin formation. Full thickness of the bronchial mucosa replaced by a neoplastic cellular proliferation characteristic of in situ SCC shows mild inflammatory reaction adjacent to the neoplastic cells. A chest X-ray showed a persistent right lower lobe mass, which was further evaluated by review of a chest CT scan (Fig. 3b), which showed a 3.5-cm right infrahilar mass that occludes the right lower lobe bronchus. Right lower lobe opacities are likely combination of mucus plugs with air space consolidation.

Microscopic examination of the right liver mass biopsy indicated HCC, the tumor comprised a trabecular pattern of hepatoid cells which were arginase positive, and showed cana- 


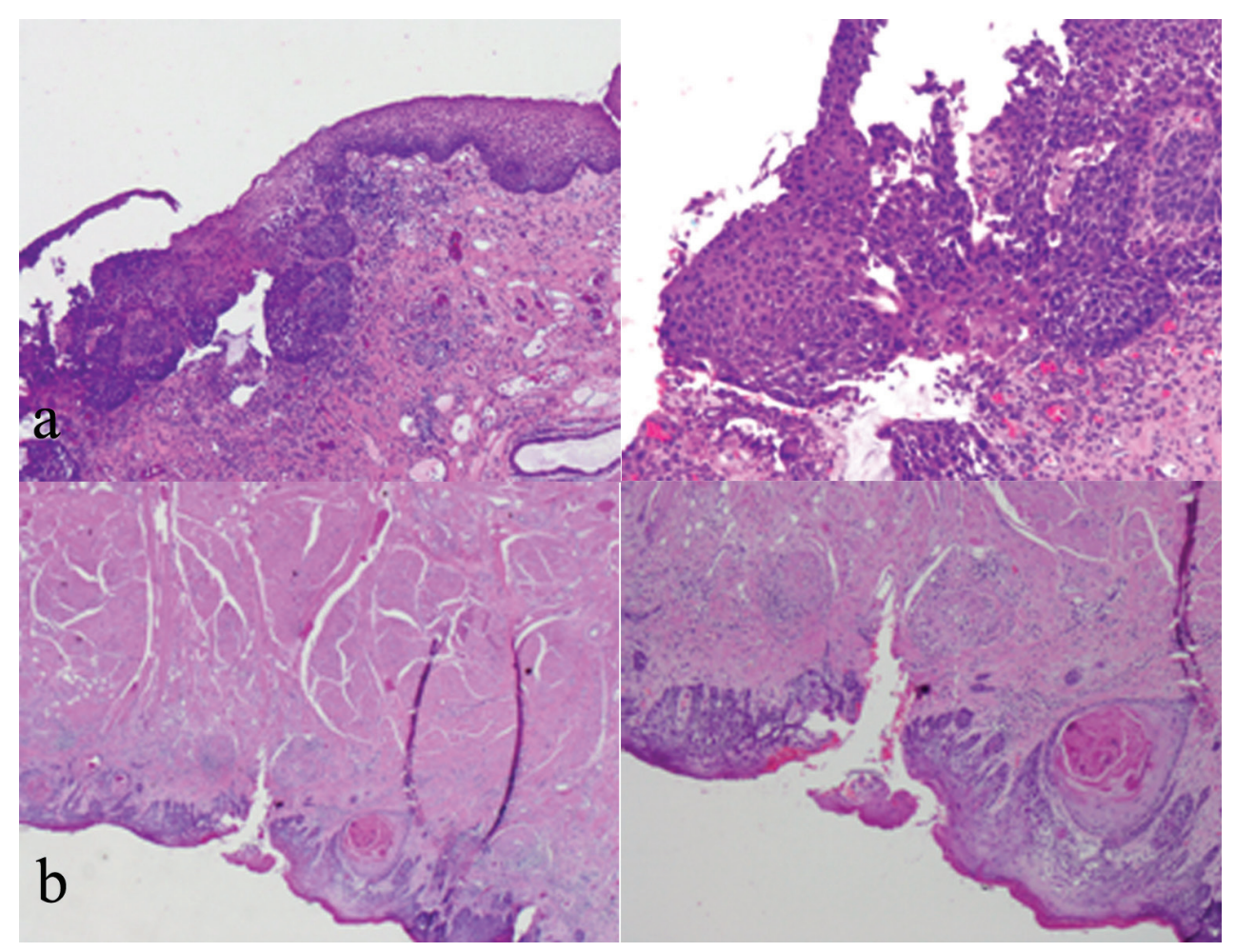

Figure 2. (a) Tongue biopsy with low and high power magnification showing violation of the basement membrane by scattered tongues of malignant epithelial cells. (b) Glossectomy microscopic examination including and revealing SCC.

liculi with a polyclonal carcinoembryonic antigen (CEA) stain (Fig. 4a). Serum studies indicated high AFP (495.4 IU/mL, normal range $0.00-6.70)$ and serum ferritin (SF, $828.3 \mathrm{ng} / \mathrm{mL}$, normal range 26 - 388), and hepatitis B e antigen and antibody were negative. All controls showed appropriate reactivity. MRI of the liver revealed T2-hyperintense mass noted in the right lobe of the liver straddling segments 5 and 8 . The mass is hypervascular on the early arterial phases and it is also proved by the evidence of washout with persistent pseudocapsule. The mass now measures $5.7 \times 4.9 \mathrm{~cm}$, producing measuring $5 \times 4$ $\mathrm{cm}$. Evidence of diffuse liver disease correlates with clinical risk factors. There was no evidence of portal vein thrombosis. No other suspicious hepatic lesions were identified (Fig. 4b). Immunohistochemistry studies were performed including CDX2, CK7, CK20, Hep Par 1, TTF-1, and P40. Hep Par 1 immunohistochemical stain in HCC shows positive cytoplasmic staining (Fig. 4c). P40 immunohistochemical stain shows no staining, helping to exclude the possibility of metastatic SCC (Fig. 4d).

According to the above test results, the patient was diagnosed with metachronous and synchronous multiple primary carcinomas in five different organs.

\section{Discussion}

Early detection and advancements in novel therapeutic modalities are improving the life expectancy of patients with neoplasm. Consequently, there is an increasing frequency of
MPCs. The National Cancer Institute (NCI) estimates that there are more than 11 million cancer survivors in the USA, more than three times the survivors in 1970 [5]. Different mechanisms have played a crucial role in the pathogenesis of PMCs, including hereditary, immune and environmental exposures such as significant cigarette smoking history, viruses, chemicals, chemotherapeutics, ionizing radiation as well as aging $[4,6]$. The prevalence and risk of MPCs increase with age, but it is not possible to identify a specific phenotype that causes increased risk of it among older individuals [7].

According to the International Agency for Research on Cancer (IARC), over 14 million new cases of cancer were diagnosed globally in 2012. The most commonly diagnosed malignant neoplasms were those of the lung and bronchus, female breast, colorectum and prostate $[8,9]$. Despite the increasing rates of MPCs, it is still considered a rare entity. The incidence rate ranges between $0.7 \%$ and $11.7 \%$ [10]. Population-based studies have attempted to estimate the incidence of a second or third malignancy in an individual that had already been diagnosed with cancer, and they found that patients with cancer were 1.29 times more likely to be susceptible of developing a new malignant tumor compared with those who were never diagnosed [11]. The risk is slightly higher for women in respect to metachronous lesions, but synchronous malignancy slightly favors men compared with women $[12,13]$.

Approximately 49,750 new cases of oral cancer are diagnosed in the USA each year. More than 9,750 people die from oral cancer each year in the USA. Oral cancer represents about $4 \%$ of all cancers and $2.2 \%$ of all cancer deaths in the 


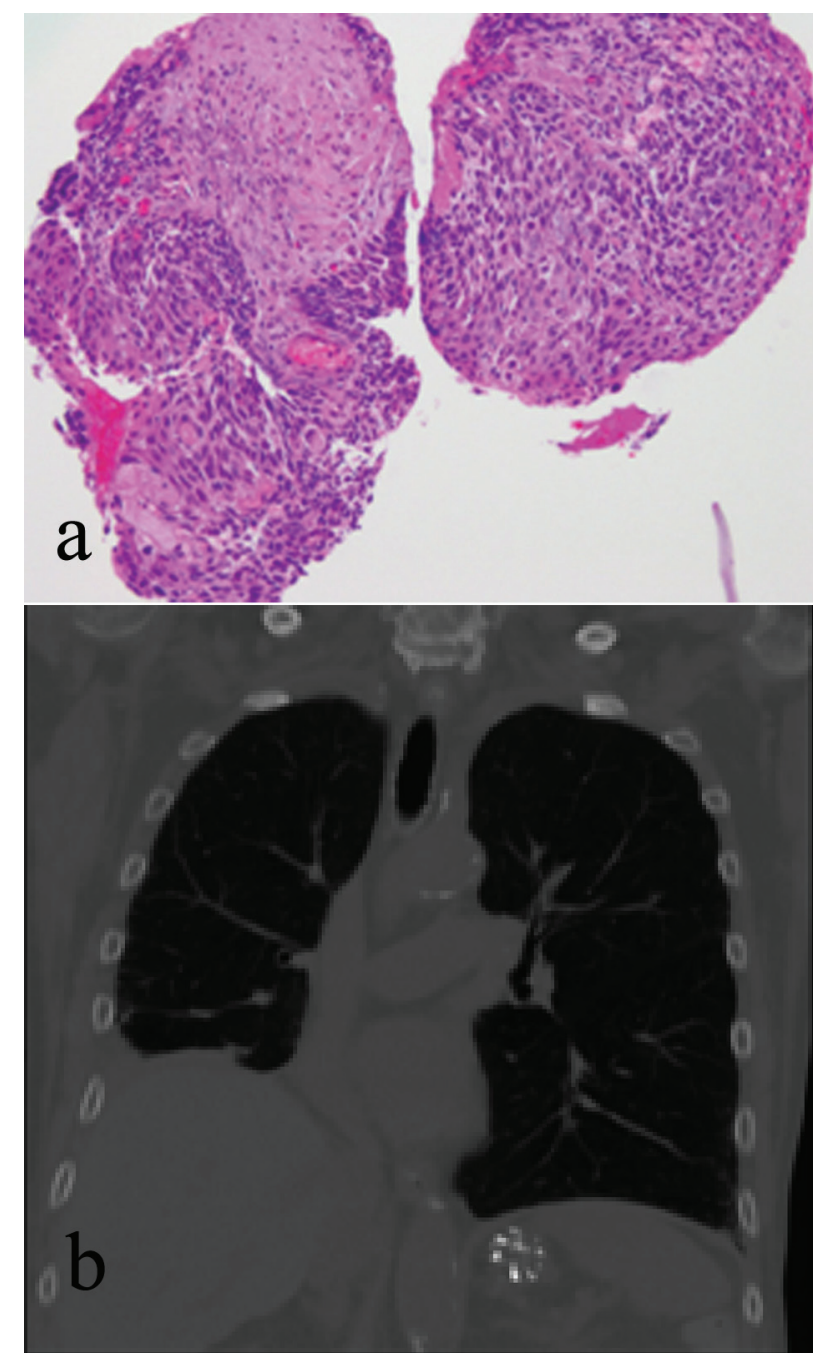

Figure 3. (a) Right lung endobronchial biopsy revealing a keratinizing SCC. (b) Chest CT scan showing a $3.5-\mathrm{cm}$ right infrahilar mass that occludes the right lower lobe bronchus.

USA [14]. Lip and oral cavity cancers accounted for nearly $17 \%$ of the total number of new cases diagnosed in $2012(2.1 \%$ globally), with two-thirds occurring in men [15]. The database of the Surveillance, Epidemiology, and End Results (SEER) analytical study reported that the primary cancers of the oral cavity, pharynx, and esophagus are strongly related to tobacco exposure and are also associated with alcohol abuse and low fruit intake. SEER-based research also found that the survivors of cancers of the oral cavity and pharynx have more than a two-fold excess risk of developing subsequent primary cancers (SPCs), and tobacco and/or alcohol consumption probably account for much of the increased risk [7, 14]. One study conducted at the University of California, San Francisco, found that more than eight out of ten oral cancer patients were smokers. For people who both smoke and drink, the risk of mouth and throat cancer increases significantly, and the combined danger is equal to or greater than the risk associated with alcohol multiplied by the risk associated with tobacco [16, 17]. The male/female ratio has decreased from 6:1 in 1950 to about
2:1 nowadays. About $95 \%$ of all oral cancers occur in persons over 40 years of age [16].

Lung cancer is the second most common malignant neoplasm. It is also the leading cause of mortality in the USA [18]. It emerged as the most common cancer worldwide several decades ago with 660,000 new cases estimated in 2012 [17]. It is the most common cancer in men (1.2 million, $16.7 \%$ of the world total) [18]. For patients who received curative surgical approach after early screening and detection, the 5-year survival rate raised up to $47-58 \%$. During follow-up time, $1.5-4.7 \%$ of lung cancer survivors developed SPCs [19, 20]. According to SEER analysis the observed annual risk (OAR) between 1973 and 2006 and absolute numbers were used to describe the risk of SPCs. The results indicate that, OAR of SPCs following lung cancer was 176.28. During follow-up, $41.26 \%$ of SPCs occurred within 12 - 59 months while the highest OAR appeared after 120 months. The overall OAR of the lung cancer after other malignancies was 27.90 [19,21]. The risk of SPCs of the lung and oral cavity is significantly high among lung cancer survivors who continue to smoke cigarettes. Some data suggest that smoking cessation following lung cancer lowers the risk of new smoking-related cancers [7, 21]. Other risk factors are passive smoking, occupational exposure to agents such as asbestos, radon, arsenic, and silica dust, air pollution and dietary factors, especially, low fruit and vegetable consumption [20]. Furthermore, OARs were higher in males than in females among all selected non-genital malignancies. These data indicated that males with such primary cancers should be screened for lung cancer more frequently than females [19,21].

The occurrence rate of liver cancer has increased dramatically in the USA during the past decade [22]. Liver cancer is the fifth commonest cancer in 2012, accounting for $9.1 \%$ of all cancer deaths globally, and the second most common cause of cancer mortality worldwide. However, liver cancer is less common in females. The ratio of liver cancer mortality to incidence is 0.95 , which means it has an extremely poor prognosis [18]. Viral hepatitis and excessive alcohol intake are the leading risk factors for the development of HCC worldwide. Some studies have reported that alcohol and tobacco may work synergistically to increase the risk of liver cancers [23]. Furthermore, chronic medical conditions such as diabetes mellitus and obesity elevate the risk of developing HCC because of the vital role the liver plays in glucose metabolism [24]. HCC occurs more often in males compared to female, with a ratio of 2:1 - 4:1. In addition to other influences, testosterone or intake of anabolic steroids has been associated with an increased occurrence of HCC and liver adenomas [25]. The risk of developing an SPC is varying at different cancer sites and is reported in a range from 1\% (primary liver malignancy) up to $16 \%$ (primary bladder cancer) [5]

Coexistence of oral cavity cancer, lung cancer and liver cancer possibly reflects the fact that certain environmental and therapeutic factors act upon these organs and this can predispose to the development of malignancies on them. The outcomes of MPCs are variable and are influenced by cancer type, affected organ and stage at diagnosis. On the other hand, genetic factors, behavioral effects, lifestyle and comorbidities generally predict patient's prognosis. In general, US African American patients have a lower incidence of multiple primaries [3]. According to Ries et al: SEER Cancer Statistics Review, 


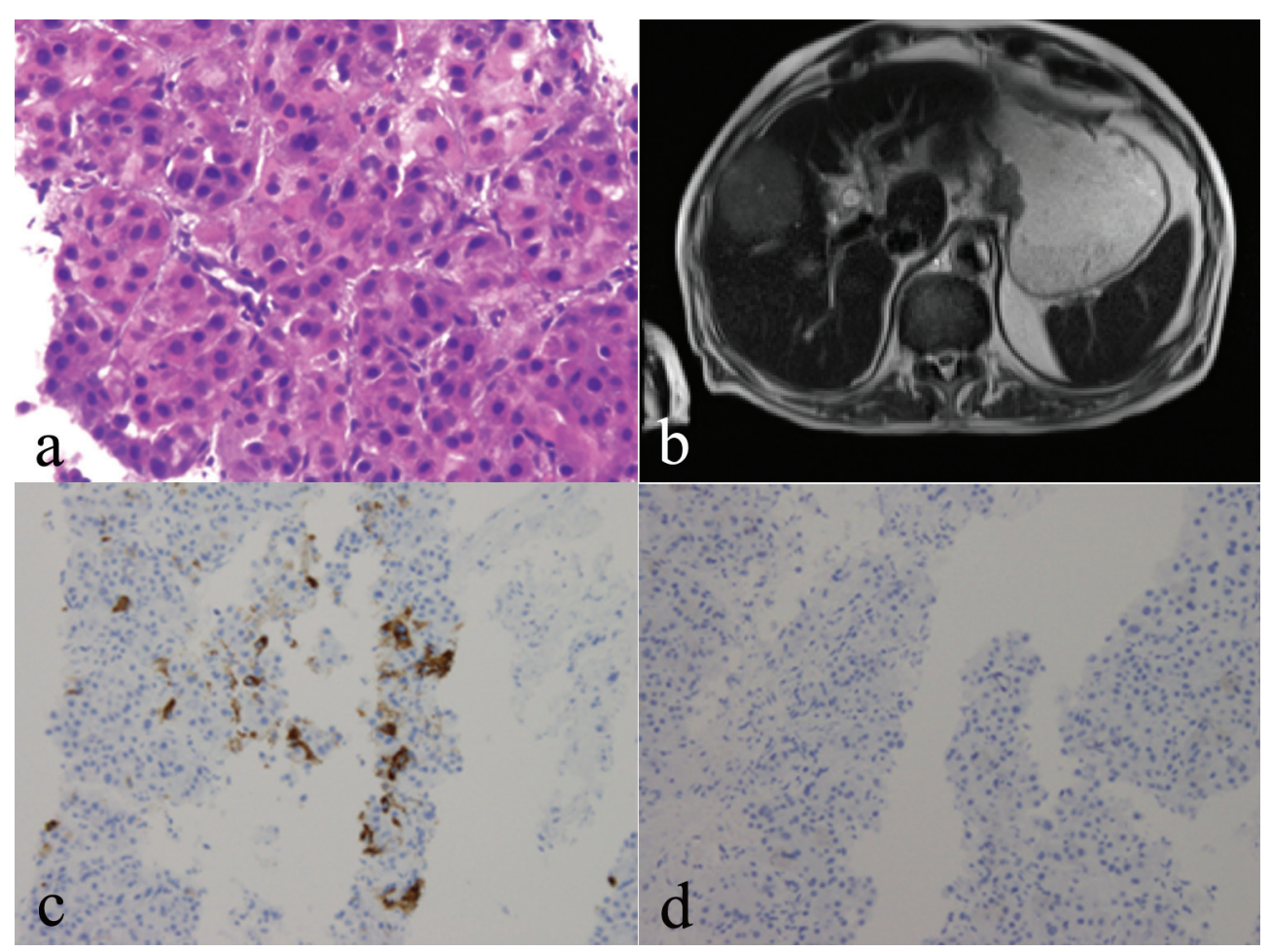

Figure 4. (a) Right liver mass biopsy indicating hepatocellular carcinoma (HCC), biopsy of which shows a trabecular growth pattern composed of thick trabeculae of neoplastic hepatocytes. Smooth contours of this group of HCC cells. Increased nuclear density, plump eosinophilic tumor cells. (b) MRI of the liver revealing T2 hyperintense mass noted in the right lobe of the liver. (c) Hep Par 1 immunohistochemical stain in HCC showing positive cytoplasmic staining. (d) P40 immunohistochemical stain showing no staining, helping to exclude the possibility of metastatic SCC.

1973 - 1995, one of the major results of an expanding aging population is an increase in the numbers of new cancer cases registered and cancer deaths recorded for the elderly population $[22,25,26]$. The analysis of this review has anticipated expansion of the older population in the next three decades in the USA. The elderly sector of population ( $>65$ years) has jumped from 12.3 million persons ( $8.1 \%$ of the population) to its present 47.8 million persons ( $14.9 \%$ of the population). In 2030 , there will be approximately one in five persons $(20.0 \%$ of the USA population) aged 65 years or older, compared with the present ratio of one in nine persons [14, 25]. Consequently, there will be an increase in the number of older persons diagnosed with multiple primary cancers $[14,26]$.

The management of patients with synchronous MPCs is challenging and should be discussed in multidisciplinary team meetings as each primary usually has a specific therapeutic strategy that might inversely influence the other primaries $[5$, 19]. Geriatric assessment (GA) has been shown to predict the risk of treatment-related complications (e.g. chemotherapy toxicity or surgical risk), but toxicity prediction at the individual level remains challenging. This is probably because individual management toxicity is dependent on an assortment of factors, including general host factors (e.g. age, genetic predisposition, and capacity for metabolizing medications), factors identified in a GA (e.g. functional status, comorbidity, nutritional condition and polypharmacy), treatment-related aspects (e.g. choice of therapeutic method, different regimens of treatments and drug-drug interactions), and tumor characteristics [26]. The patient should be informed about this difficult situation and limitation of therapeutic approaches with uncertain prognosis. It is important that tumor survivors follow the recommendations for cancer prevention and early detection, including tobacco cessation, physical activity, healthy diet, weight control, and if applicable cancer screening and surveillance for recurrence [19].

\section{Conclusions}

The occurrence of cancer rises with age. Many more lifesaving treatments are being offered to older adults, as newer treatments are often better tolerated and have less impact on quality of life. Due to the dramatic increase in the population of both healthy older adults, as well as those who have survived previous, serious medical illness, we can expect to see an increase in MPCs in this vulnerable population. In this report, we described a case of a man with the combination of oral cavity, lung and liver MPCs. It is important for health care providers to consider the possibility of the appearance of subsequent, primary malignant neoplasm in a cancer patient. This is especially important for the one who has an extensive history of tobacco use. The probability of a metachronous or a synchronous malignancy should be evaluated with regular and prolonged follow-up to improve the outcomes. 


\section{Conflict of Interest}

None.

\section{Funding}

None.

\section{References}

1. Li F, Zhong WZ, Niu FY, Zhao N, Yang JJ, Yan HH, Wu YL. Multiple primary malignancies involving lung cancer. BMC Cancer. 2015;15:696.

2. Warren S, Gates O. Multiple primary malignant tumors: a survey of the literature and statistical study. Am J Cancer. 1932; $16: 1358-1414$.

3. Vogt A, Schmid S, Heinimann K, Frick H, Herrmann C, Cerny T, Omlin A. Multiple primary tumours: challenges and approaches, a review. ESMO Open. 2017;2(2):e000172.

4. Soerjomataram I, Coebergh JW. Epidemiology of multiple primary cancers. Methods Mol Biol. 2009;471:85105.

5. www.cancer.org/content/dam/cancer-org/research/ cancer-facts-and-statistics/annual-cancer-facts-and-figures/2009/special-section-multiple-primary-cancers-cancer-facts-and-figures-2009pdf.

6. Amer MH. Multiple neoplasms, single primaries, and patient survival. Cancer Manag Res. 2014;6:119-134.

7. Weir HK, Johnson CJ, Ward KC, Coleman MP. The effect of multiple primary rules on cancer incidence rates and trends. Cancer Causes Control. 2016;27(3):377-390.

8. Forman D, Ferlay J. World cancer Report 2014. In: Stewart BW, Wild CP, editors; The global and regional burden of cancer. Vol. 2014. International Agency for Research on Cancer; Lyon, Rance: 2014.

9. Bray F, Jemal A, Grey N, Ferlay J, Forman D. Global cancer transitions according to the Human Development Index (2008-2030): a population-based study. Lancet Oncol. 2012;13(8):790-801.

10. Coleman MP. Multiple primary malignant neoplasms in England and Wales, 1971-1981. Yale J Biol Med. 1986;59(5):517-531.

11. Schoenberg BS. Multiple primary malignant neoplasms. The Connecticut experience, 1935-1964. Recent Results Cancer Res. 1977;58:1-173.

12. Spratt JS, Jr., Hoag MG. Incidence of multiple primary cancers per man-year of follow up: 20-year review from the Ellis Fischel State Cancer Hospital. Ann Surg. 1966;164(5):775-784.

13. Sakellakis $M$, Peroukides $\mathrm{S}$, Iconomou G, Boumpoucheropoulos S, Kalofonos $\mathrm{H}$. Multiple primary malignancies: a report of two cases. Chin J Cancer Res. 2014;26(2):215-218.

14. Caporaso NE, Dodd KW, Tucker MA. New malignan- cies following cancer of the respiratory tract. In: Curtis RE, Freedman DM, Ron E, et al., eds. New Malignancies Among Cancer Survivors: SEER Cancer Registries, 1973-2000, 2006. Bethesda, MD p. 145-180. (NIH Publ. No. 05-5302; Available at http://seer.cancer.gov/publications).

15. Ferlay J, Jacques Ferlay, Isabelle Soerjomataram, Rajesh Dikshit, Sultan Eser, Colin Mathers, Marise Rebelo Donald Maxwell Parkin, David Forman, Freddie Bray. First published: 13 September 2014. GLOBOCAN 2012 v1.0, Cancer Incidence and Mortality Worldwide. IARC Cancer Base No. 11. Lyon, France: International Agency for Research on Cancer. 2013.

16. Cao Y, Willett WC, Rimm EB, Stampfer MJ, Giovannucci EL. Light to moderate intake of alcohol, drinking patterns, and risk of cancer: results from two prospective US cohort studies. BMJ. 2015;351:h4238.

17. Negri E, La Vecchia C, Franceschi S, Tavani A. Attributable risk for oral cancer in northern Italy. Cancer Epidemiol Biomarkers Prev. 1993;2(3):189-193.

18. Maruyama T, Nakasone T, Maruyama N, Matayoshi A, Arasaki A. Synchronous quadruple multiple primary cancers of the tongue, bilateral breasts, and kidney in a female patient with a disease-free survival time of more than 5 years: a case report. World J Surg Oncol. 2015;13:263.

19. Demark-Wahnefried W, Pinto BM, Gritz ER. Promoting health and physical function among cancer survivors: potential for prevention and questions that remain. J Clin Oncol. 2006;24(32):5125-5131.

20. Chuang SC, Scelo G, Lee YC, Friis S, Pukkala E, Brewster DH, Hemminki $\mathrm{K}$, et al. Risks of second primary cancer among patients with major histological types of lung cancers in both men and women. Br J Cancer. 2010;102(7):1190-1195.

21. Mao R, Chen T, Zhou F, Jiang W, Yang X, Ai Z, Li M, et al. The burdens of lung cancer involved multiple primary cancers and its occurring patterns-SEER Analysis between 1973 and 2006. Sci Rep. 2017;7(1):6451.

22. Kuper H, Tzonou A, Kaklamani E, Hsieh CC, Lagiou P, Adami HO, Trichopoulos D, et al. Tobacco smoking, alcohol consumption and their interaction in the causation of hepatocellular carcinoma. Int J Cancer. 2000;85(4):498502.

23. Marrero JA, Fontana RJ, Fu S, Conjeevaram HS, Su GL, Lok AS. Alcohol, tobacco and obesity are synergistic risk factors for hepatocellular carcinoma. J Hepatol. 2005;42(2):218-224.

24. Wang C, Wang X, Gong G, Ben Q, Qiu W, Chen Y, Li $\mathrm{G}$, et al. Increased risk of hepatocellular carcinoma in patients with diabetes mellitus: a systematic review and meta-analysis of cohort studies. Int $\mathrm{J}$ Cancer. 2012;130(7):1639-1648.

25. White DL. Epidemiology of hepatocellular carcinoma. In: Carr BI, editor. Hepatocelluar Carcinoma. New York, NY: Humana Press; 2010. pp. 1-25.

26. Balducci L, Lyman GH. Cancer in the elderly. Epidemiologic and clinical implications. Clin Geriatr Med. 1997;13(1):1-14. 